\title{
MR Navigated Breast Surgery: Method and Initial Clinical Experience
}

\author{
Timothy Carter ${ }^{1}$, Christine Tanner ${ }^{1}$, Nicolas Beechey-Newman ${ }^{2}$, \\ Dean Barratt ${ }^{1}$, and David Hawkes ${ }^{1}$ \\ ${ }^{1}$ Centre for Medical Image Computing, University College London, WC1E 6BT, UK \\ \{timothy.carter, c.tanner, d.barratt, d.hawkes\}@ucl.ac.uk \\ ${ }^{2}$ Hedley Atkins Breast Unit, Guy's Hospital, London, SE1 9RT, UK \\ nicolas.beechey-newman@gstt.nhs.uk@cs.ucl.ac.uk
}

\begin{abstract}
D dynamic contrast enhanced magnetic resonance (MR) images may help to reduce the high re-excision rate associated with breast conserving surgery. However these images are acquired prone, whilst surgery is performed supine which results in a large deformation that limits their usefulness. We describe here a registration technique based on a biomechanical model to account for soft tissue deformation between prone MR imaging and surgery. The accuracies of the individual registration steps are assessed off-line. We then report our first clinical experience with an image-guided surgery system which incorporates these algorithms. The system's accuracy is assessed against tracked ultrasound images, and is determined to be around $5 \mathrm{~mm}$ for this case.
\end{abstract}

\section{Introduction}

The most common treatment for breast cancer involves excising the cancer and a small volume of surrounding healthy tissue, but conserving the majority of the breast tissue. A disadvantage of this procedure is that a large proportion of these operations need to be repeated because, on histological examination, cancer is found at the margins of the surgical specimen indicating that it has not been completely excised.

The majority of cancers are located by palpation, but this is not possible for cancers such as ductal carcinoma in situ which are no stiffer than the surrounding tissue, and the absence of distinct tissue boundaries within the breast makes the complete excision of diffuse cancers and small lesions challenging. For impalpable lesions the surgeon relies upon a guidewire inserted preoperatively under ultrasound guidance and upon X-ray mammograms to help him predict the extents of the cancer. Relating the single point identified by a guidewire and the 2D projection images of the compressed breast provided by mammograms to the $3 \mathrm{D}$ extents of the cancer during surgery is not straightforward. Furthermore, the palpable extents of a lesion do not necessarily correlate well with histology findings.

Dynamic contrast-enhanced (DCE) magnetic resonance (MR) images provide additional 3D information about the location of a cancer. However, a large deformation of the breast will occur between acquiring these images in the prone position (to reduce breathing motion between images acquired at different timepoints) and performing 
the operation in the supine position. This currently limits their suitability for guiding surgery.

Previous work [1] has outlined a framework for image-guided breast surgery which uses a biomechanical model to link preoperative MR imaging with surgery. In this paper we develop the framework, focusing on presenting an improved biomechanical model of the deformation and on assessing the registration on a greater number of subjects. We conclude by reporting our experiences of what we believe to be the first operation in which an image-guidance system was used to non-rigidly register preoperative MR images with the surgical scene in order to locate breast cancer.

\section{Registration Method}

To simplify the task of recovering the deformation between prone imaging and surgery a supine MR image is acquired without contrast enhancement and the registration is divided into two stages. In the preoperative stage the cancer identified in the prone DCE MR image is located in the supine image, which allows intensity information to be used to help guide the registration. During the intraoperative stage a biomechanical model constructed from the supine MR image is used to recover the smaller deformation between supine imaging and surgery.

\subsection{MR Imaging and Preoperative Registration}

MR Imaging. Self-adhesive MR-visible fiducial markers were attached to the skin surface during all MR imaging. Their positions were marked with ink on the skin in order that these locations were also identifiable during surgery. DCE MR images were acquired with the patient in a prone position using a breast coil, according to the hospital's standard clinical protocol. These images included a coronal dataset acquired pre-contrast using a 3D gradient echo sequence which is used for the prone-supine registrations described in this paper. In addition an axial image was acquired in the supine position using a 3D gradient echo sequence. The body coil was used for supine imaging so that no mechanical forces were applied to the surface of the breast.

Overview of Preoperative Registration Technique. As can be seen in Figure 1, because the deformation between the prone and supine positions is large there will be a poor initial image overlap. This meant that standard intensity-based registration techniques, such as [2] and [3], were found to be inadequate since the algorithms become trapped in local minima. Therefore a two step 'hybrid' registration technique is used to locate the cancer in the supine image. In the first step a finite element model is used to predict the deformation which the breast undergoes between the prone and supine positions. In the second step a simulated MR image is generated from the supine image using the model deformations. This image has an improved overlap with the prone image, and so an intensity-based registration algorithm can be used to match the images.

Constructing the Finite Element Mesh. Initial experimentation showed that the hexahedral elements were less susceptible than tetrahedral elements to becoming 
badly shaped when undergoing large gravity-induced deformations. Therefore the supine MR image of the breast was meshed into hexahedral elements. These elements were created by meshing each slice of the MR image as shown in Figure 1 and then connecting the adjacent 2D meshes to form a 3D mesh. Elements were assigned a material type of either adipose or fibroglandular tissue depending on the predominant material type contained within the element, and densities of 928 and $1035 \mathrm{kgm}^{-3} \mathrm{re}-$ spectively [4] were assigned to the two tissue types

Modelling the Deformation Between Supine and Prone. The finite element analysis used in [1] assumed infinitesimal strain when modelling the deformation between prone and supine, and so all integrations were performed over initial element shapes. The deformation of the breast between the prone and supine positions is large, and so this is not a good approximation. In this work we use a finite strain formulation which takes the deforming geometry into account. A mixed u-P element formulation is used to enforce incompressibility. All biomechanical modeling in this paper is performed using the ANSYS finite element software (ANSYS Inc., Canonsburg, PA, USA).
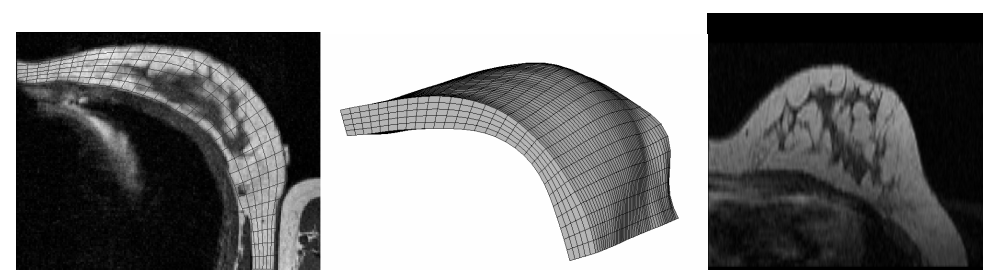

Fig. 1. (left) Transverse section through supine breast overlaid with finite element mesh (centre) finite element model in supine position (right) breast in prone position.

To correctly model the deformation between the prone and supine positions, it is necessary to recognise that gravity was acting on the breast when the supine MR image was acquired and so unknown loads exist within the supine model [5]. An iterative method was used here to obtain the unloaded reference state. Firstly, an initial estimate of the reference state was constructed by applying gravity in the anterior direction, and setting the stresses within the model to zero. Gravity was then applied in the posterior direction on the reference state model. If the reference state is accurate the nodes of the deformed reference model will be coincident with the nodes of the supine model. Otherwise the nodes of the reference state model can be appropriately adjusted and gravity applied once again. The process is repeated until the deformed reference model nodes and the supine reference nodes are coincident to within a tolerance (chosen to be $0.05 \mathrm{~mm}$ ). Gravity is then applied in the anterior direction on the reference state model.

Experiments to determine the material properties of the breast (e.g. [6] [7]) have only considered relatively small compressions. There is no data available which is appropriate for deformations of the scale considered here, or for the breast in extension. Using material properties from the literature results in a deformation which is much smaller than is actually observed and so in this paper the properties are instead determined by fitting the modelled deformation to the observed deformation. To 
achieve this, fibroglandular and adipose tissue are assumed to obey the same neoHookean relationship and the value of the initial shear modulus is iteratively adjusted until the distance between the chest wall and the nipple in the model agrees with this distance measured in the prone MR image.

Examination of pairs of prone-supine MR images indicates that there is significant 'sliding' of the breast around the torso, and failing to account for this results in a simulated prone breast which is narrower in the medio-lateral direction than is actually observed. This sliding motion was included in the model by applying displacement boundary conditions on nodes of the posterior face of the model which moved the nodes along this face. The distance moved by the nodes increased linearly with the distance along the face from the midline, so that the most medial nodes remained stationary whilst the most lateral nodes moved furthest. The appropriate level of sliding was determined by identifying corresponding features close to the pectoral wall in the prone and supine images. The same boundary conditions were applied to each node of the lateral face that had applied corresponding node lying on the posterior face. The medial face was constrained to be stationary. Gravity was modelled as a body force equivalent to an acceleration of $9.81 \mathrm{~ms}^{-2}$.

Surface Alignment of Model with Prone MR. The correspondence between the model and the prone MR image was further improved by deforming the model such that its skin surface aligned with the surface in the MR image. The positions of the fiducial markers in the deformed model were calculated, and the marker locations used to rigidly align the model with the prone MR image. A dense cloud of points lying on the skin surface of the prone MR image was extracted by automatically thresholding the image, finding the crossing point between air and tissue and smoothing this surface. Displacement boundary conditions were then imposed upon each of the nodes on the skin surface of the model in the direction normal to the surface. The displacements were equal to half of the distance to the closest point on the prone skin surface, resolved into the normal direction for the initial iteration when the surfaces are not well aligned, and equal to this value for all further iterations. No constraints were imposed upon the displacement of nodes in the tangential directions. The rigid alignment and model deformation steps were repeated until the surfaces aligned to within a tolerance of $0.1 \mathrm{~mm}$.

Intensity-Based Registration. MR images were corrected for image inhomogenity [8]. The displacements of the finite element nodes were used to deform the supine image to an image which approximately matched the prone image. Both images were resampled to have isotropic voxels with an edge length equal to the in-plane voxel dimension of the original supine MR image. These two images were then registered using a fluid registration algorithm [2] in which the transformation between two images is modelled as the flow of a compressible viscous fluid [9]. The model deformed supine image was treated as the target image, and an image force of zero was applied to all voxels lying outside the deformed model. Normalised intensity cross correlation was chosen as the image similarity measure. The regridding threshold (the fractional change in voxel volume below which the current transformation is applied and the algorithm restarted) was set to 0.5 . A stopping condition based on the change in 
similarity measure at each iteration was used to terminate the registration when there was no further improvement. On the basis of initial experiments, the algorithm was run at a quarter image resolution.

The lesion was segmented from subtraction images formed from the prone DCE MR images. A triangulated surface was generated from this segmentation, and the hybrid registration formed by combining the displacements of the biomechanical model and fluid registration steps was applied to the vertices of the triangulation to locate the cancer within the supine MR image and model.

\subsection{Intraoperative Registration}

During surgery the patient was positioned supine, with her arm on the involved side abducted perpendicularly. A stereo camera system (Vision RT, London, UK) was positioned at the end of the arm, at a range of approximately $1.5 \mathrm{~m}$ from the patient's breast. Images were reconstructed of the surface of the breast before the first incision. The 3D skin surface output by the stereo camera consists of around 3500 points with approximately a $3.5 \mathrm{~mm}$ separation. The fiducial marker locations were identified using a texture image aligned with this surface.

The position of the cancer in the supine finite element model was calculated in Section 2.2. The same model is used to compute the position of cancer during surgery. The fiducial marker locations were used to align the finite element model with the stereo camera surface. Displacement loads were then applied on the node closest to each fiducial marker such that the node became coincident with the homologous fiducial marker location in the stereo camera image. Displacement loads were applied on all other nodes lying on the skin surface of the model in the direction normal to the skin surface, and with a magnitude equal to the distance from the node to the closest point on the skin surface projected onto the normal direction. These nodes were not constrained in the direction tangential to the skin surface. In order that the results were available within a surgically useful timeframe of about one minute, and because this deformation was small, this one-step approach was adopted rather than the iterative approach adopted for matching surfaces described in the previous section. For the same reasons, the breast was assumed to be a homogenous linear elastic material, and infinitesimal strain was assumed. Incompressibility was modelled by assuming a Poisson's ratio of 0.495 .

\section{Registration Assessment}

The accuracy of the prone-supine registration described in Section 2.2 was assessed for three subjects on eight corresponding landmarks identified in the prone and supine MR volumes. The results are summarized in Figure 2 which shows that for this task the registration accuracy of the hybrid registration technique is a marked improvement over both rigid registration and the fluid registration technique described in [2]. Transverse slices through a supine MR volume and the corresponding prone volume deformed according to the hybrid registration are shown in Figure 3 for an example dataset. The registration appears visually reasonable. 


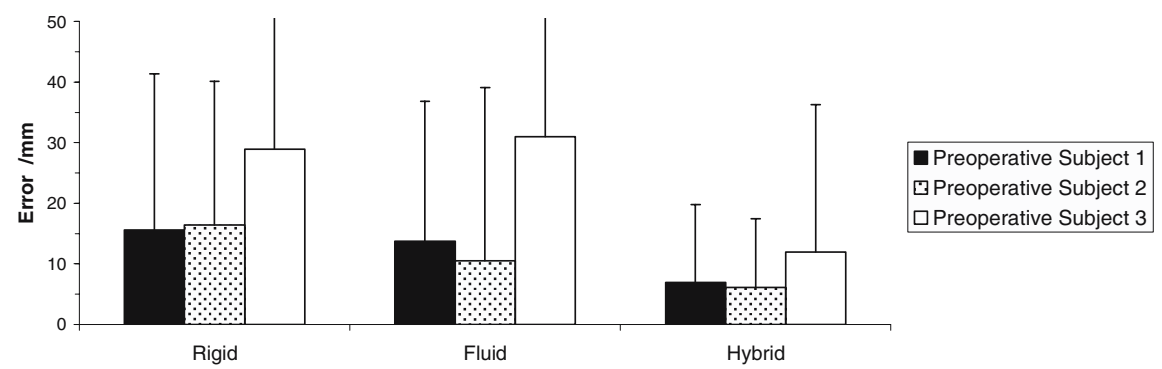

Fig. 2. Mean preoperative registration errors using rigid, fluid and hybrid registration techniques. The maximum registration error is indicated by the error bars, which have been truncated for Subject 3 (rigid) and Subject 3 (fluid) since they exceed 50mm.
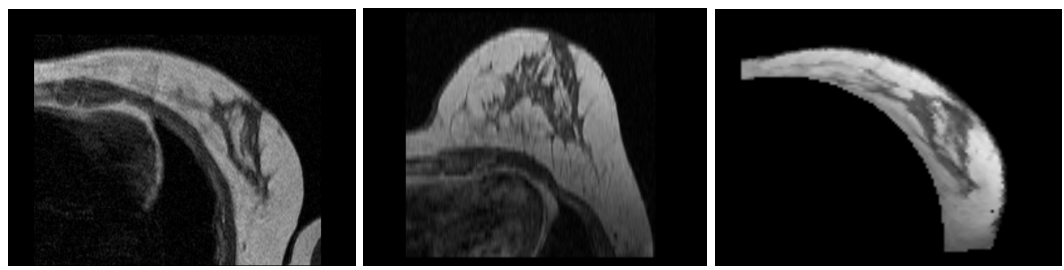

Fig. 3. (left) Example transverse section through supine MR image (centre) approximately corresponding transverse section through prone MR image (right) corresponding transverse section through an image formed by applying the registration deformation field to the prone MR image

The intraoperative registration technique described in Section 2.3 was assessed for two subjects by simulating the intraoperative surface from an additional supine MR image. Each subject sat up after the first image was acquired so the pairs of supine images were noticeably displaced. The ability of the model to recover the deformation was assessed on eight pairs of corresponding features identified in the images. The model-based registration accuracies are compared in Table 1 with the registration accuracies achieved by rigidly registering the fiducial marker locations.

Table 1. Accuracy of intraoperative registration technique

\begin{tabular}{lccc}
\hline & Mean (max) rigid error $/ \mathrm{mm}$ & Mean (max) non-rigid error $/ \mathrm{mm}$ \\
\hline Intraoperative Subject 1 & $3.2(5.1)$ & $1.9(3.0)$ \\
Intraoperative Subject 2 & $4.9(8.8)$ & $2.6 \quad(5.1)$ \\
\hline
\end{tabular}

\section{Initial Clinical Experience}

The system has been demonstrated in the operating theatre on a 47 year old patient with a palpable lesion in the upper outer quadrant of her left breast. The lesion was felt clinically to be very peripheral with a diameter of $18 \mathrm{~mm}$, and ultrasound showed a lesion of diameter $20 \mathrm{~mm}$. However, when DCE MR imaging was performed seven 
days before the operation it indicated that the lesion had a much larger diameter of $35 \mathrm{~mm}$ and that it extended right down to the pectoral muscle.

The position of the lesion, as indicated in the prone DCE MR images, was calculated in the supine model prior to the operation. An image-guidance system incorporating the registration techniques described in this paper was used to indicate the position of the lesion after the patient had been stabilised on the operating table, immediately prior to sterilization. Deforming the finite element model to update the lesion location took 45 seconds. The lesion was presented to the surgeon as a surface rendered underneath a semi-transparent skin surface (Figure 4) which could be rotated to match the surgeon's viewpoint. Live video was overlaid on the rendered skin surface so that outlines drawn on the skin could be seen on both the patient and the rendered surface. The image-guidance system also included a tracked pointer.

To assess the system accuracy, the lesion was also imaged using an ultrasound probe that was tracked using an Optotrak system (Northern Digital Inc., Ontario, Canada). Care was taken to deform the breast minimally whilst imaging. Since the breast was not being compressed, it was not possible to image the deep margin of the lesion. The positions of the fiducial markers on the surface of the breast were recorded using a tracked pointer, and so the ultrasound images could be rigidly aligned with the model predictions, as shown in the example ultrasound slice of Figure 4. A good match between the ultrasound images and the lesion boundaries predicted from MR is observed, with the margin identified in the two modalities agreeing to within about $5 \mathrm{~mm}$ where visible. The registration is perhaps better than might be expected given the preoperative registration errors calculated in Section 3. This may be explained by the boundaries of the lesion coinciding with a strong fibroglandular-toadipose tissue boundary, which caused a more accurate prone-supine registration in this region.
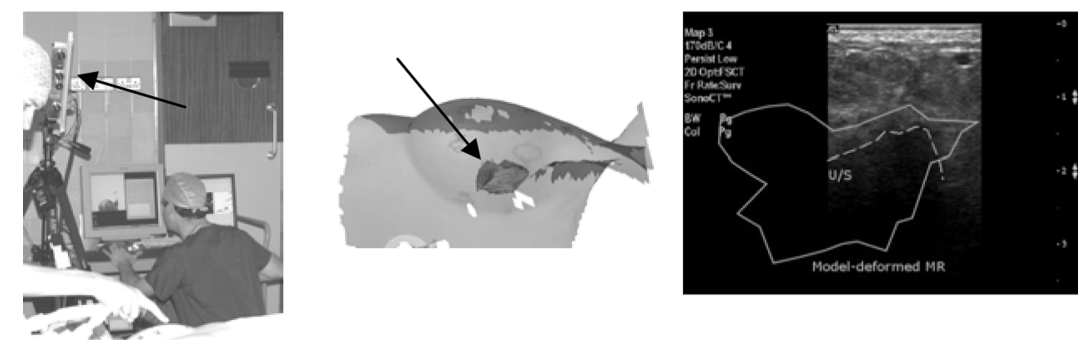

Fig. 4. (left) Image-guidance system in the operating theatre. The patient is just visible in foreground and the stereo camera is indicated with an arrow. (centre) Surface rendering of breast as presented during surgery with video overlay. Lesion is indicated with an arrow. (right) predicted lesion location (solid line) overlaid on ultrasound image showing lesion (dashed line).

A large surgical excision was performed. On histological examination the lesion was found to have a maximal dimension of $35 \mathrm{~mm}$ and to extend to within $2 \mathrm{~mm}$ of the pectoral muscle, which is much more consistent with the lesion location indicated by the image-guided surgery system than with the palpable mass that could be felt by the surgeon. 


\section{Conclusion}

Image-guided surgery based on DCE MR images may help to reduce the re-excision rate associated with breast-conserving surgery. This paper has presented and assessed the registration steps required to transform the location of the lesion from the prone MR images into the surgical space. In particular the large deformation between prone and supine is modeled using a finite strain formulation and taking into account the fact that the breast is gravity-loaded when imaged. The hybrid registration approach to recovering the prone-supine deformation presented here was shown to be twice as accurate as a widely used intensity-based registration algorithm for this task. It was also shown that a biomechanical model can be used to estimate the displacement occurring between a supine MR image and surgery driven only by displacements applied to the exterior surface of the model.

The first breast surgery case in which an image-guidance system deformed preoperative images to match the surgical scene has been reported. Comparison with tracked ultrasound images suggests that for this case the system was accurate to around $5 \mathrm{~mm}$. Since a surgeon will typically aim to remove a $5-10 \mathrm{~mm}$ margin of healthy tissue surrounding a cancer in order to allow for the inaccuracies inherent in current techniques, image-guided breast surgery has the potential to become a surgically useful tool.

\section{References}

1. Carter, T.J., Tanner, C., Crum, W.R., Beechey-Newman, N., Hawkes, D.J.: A Framework for Image-Guided Breast Surgery. In: Yang, G.-Z., Jiang, T., Shen, D., Gu, L., Yang, J. (eds.) MIAR 2006. LNCS, vol. 4091, pp. 203-210. Springer, Heidelberg (2006)

2. Crum, W.R., Tanner, C., Hawkes, D.J.: Multiresolution Anisotropic Fluid Registration: Evaluation in Magnetic Resonance Breast Imaging. Physics in Medicine and Biology 50, 5153-5174 (2005)

3. Rueckert, D., Sonoda, L.I., Hayes, C., Hill, D.L., Leach, M.O., Hawkes, D.J.: Non-rigid Registration using Free-Form Deformations: Application to Breast MR Images. IEEE Transactions on Medical Imaging 18, 712-721 (1999)

4. Johns, P.C., Yaffe, M.J.: X-ray characterisation of normal and neoplastic breast tissue. Physics in Medicine and Biology 32, 675-695 (1987)

5. Pathmanathan, P., Gavaghan, D., Whiteley, J., Brady, M., Nash, M., Nielsen, P., Rajagopal, V.: Predicting Tumour Location by Simulating Large Deformations of the Breast Using a 3D Finite Element Model and Nonlinear Elasticity. In: Barillot, C., Haynor, D.R., Hellier, P. (eds.) MICCAI 2004. LNCS, vol. 3217, pp. 217-224. Springer, Heidelberg (2004)

6. Krouskop, T.A., Wheeler, T.M., Kallel, F., Garra, B.S., Hall, T.: Elastic Moduli of Breast and Prostate Tissues Under Compression. Ultrasonic Imaging 20, 260-274 (1998)

7. Samani, A., Zubovits, J., Plewes, D.: Elastic moduli of normal and pathological human breast tissues: an inversion-technique-based investigation of 169 samples. Physics in Medicine and Biology 52, 1565-1576 (2007)

8. Sled, J.G., Zijdenbos, A.P., Evans, A.C.: A non-parametric method for automatic correction of intensity non-uniformity in MRI data. IEEE Transactions on Medical Imaging 17, 87-97 (1998)

9. Christensen, G.E., Rabbitt, R.D., Miller, M.I.: Deformable templates using large deformation kinematics. IEEE Transactions on Image Processing 5, 1435-1447 (1996) 